\title{
DECISION PROCEDURES FOR FAULT DETECTION AND ISOLATION DERIVED FROM KNOWLEDGE AND DATA
}

\author{
A. Evsukoff ${ }^{1}$, P. Weber and S. Gentil \\ Laboratoire d'Automatique de Grenoble, UMR CNRS-INPG (ENSIEG) -UJF, \\ BP 46, 38402 Saint Martin d'Hères, France; Phone : (+33) 476826413 - Fax : (+33) 476826388 \\ e-mail : evsukoff@lmp.ufrj.br ; Philippe.Weber@inpg.fr ; Sylviane.Gentil@inpg.fr
}

Keywords: Fault Detection and Isolation, Fuzzy Systems, Knowledge Based Systems, Supervised Learning.

\begin{abstract}
This work presents a unified approach to derive decision procedures for model based fault detection and isolation (FDI) either from knowledge or from experiments. In the knowledge-based approach, fuzzy rule weights are defined directly from model structure. In the supervised learning approach, the decision procedure is derived from a data set. The symbolic to numeric integration provided by fuzzy sets in the proposed framework allows integrating symbolic symptoms into the decision procedure. The proposed method is applied to the FDI of a winding machine.
\end{abstract}

\section{Introduction}

In model based fault detection and isolation (FDI) methods, residuals are fault indicators that express the consistency between the model and the actual behaviour of the process. A first theoretical step consists in generating relevant residuals, that allow detecting the faults in a manner robust to other system perturbations. A second more practical step consists in deciding on line to which faulty situation the residuals correspond.

Most of the residual generation methods use estimation techniques, such as observers or parity space approaches [Isermann 97]. These methods are closely related. A linear transformation of the original residuals improves fault isolation by decoupling the effects of faults from measurable disturbances and modelling errors.

On line FDI is provided by a decision step that can be performed by different approaches: Dempster-Shafer evidential reasoning [Gertler 92], logic voting schemes [Cassar 92] or fuzzy logic approaches [Montmain 91];

${ }^{1}$ Present adress : PEN/COPPE-UFRJ, Laboratório de Monitoração de Processos, POBox 68509, 21945-970 - Rio de Janeiro, RJ, Brasil
[Frank 94]; [Isermann 98]. This paper is devoted to this problem. Most methods are based on the structure of the models used for residual generation.

In large-scale industrial applications, models are commonly based on heuristics or experimental data and model structure may not be clearly defined. Additionally, fault diagnosis may take into account symbolic information that cannot be directly integrated into mathematical models. In such cases, analytical decoupling is not possible, but the design procedure can be derived from data.

In this work, a general framework based on fuzzy systems is proposed so that the FDI decision procedure can be designed either from knowledge of the model structure or derived from data. The paper is organised as follows. In the second section, the general framework for fuzzy relations and decision making is introduced. Section 3 presents the decision procedure design based on the knowledge of model structure or derived from data. In section 4, the proposed method is applied to the FDI of a winding machine; this simple example is used to appreciate how FDI can be derived from data.

\section{Fuzzy systems for decision-making}

\subsection{Preliminaries}

A fuzzy set is denoted by:

$$
\tilde{A}=\{(x, A(x)), x \in X\}
$$

where $X$ is the reference domain, generally a subset of real numbers, and $A: X \mapsto[0,1]$ is the membership function.

The elements of $X$ are described by the symbolic terms in the descriptor set $\mathbf{A}(X)$. Each symbolic term $A_{i} \in \mathbf{A}(X)$ represents a generalisation of the reference values whose meaning is the fuzzy set $\tilde{A}_{i}$. In practical applications, terms $A_{i} \in \mathbf{A}(X)$ have a linguistic interpretation and the set of all meanings $\tilde{A}_{i}$ forms a fuzzy partition of the reference domain. 
For every element $x_{0} \in X$, its description $\tilde{X}_{0}$ is a fuzzy set defined on $\mathbf{A}(X)$. The description $\tilde{X}_{0}$ can be represented by a line vector, defined in the unit cube $I^{n}$ [Kosko 92], where $n$ is the cardinality of $\mathbf{A}(X)$ and:

$$
\tilde{X}_{0}=\left[X_{0}\left(A_{1}\right) \ldots X_{0}\left(A_{n}\right)\right]=\left[A_{1}\left(x_{0}\right) \ldots A_{n}\left(x_{0}\right)\right]
$$

Fuzzy descriptions can be related by a set of rules to describe a linguistic model between several reference domains. Such models are implemented by fuzzy systems, described next.

\subsection{Symbolic Fuzzy Systems}

A linguistic model relating variables $x$ and $y$ can be written by a rule base relating the terms in the descriptor sets $A_{i} \in \mathbf{A}(X)$ and $B_{j} \in \mathbf{B}(Y)$, with rules like:

$$
\text { if } x \text { is } A_{i} \text { then } y \text { is } B_{j} \text {. }
$$

The rule base is a collection of rules like (3) and can be represented by the symbolic fuzzy relation $\tilde{\Phi}$ in the cartesian product $\mathbf{A}(X) \times \mathbf{B}(Y)$ [Foulloy 95]. Rules in the rule base are weighted by the membership value $\Phi\left(A_{i}, B_{j}\right)$ that represents how much the term $A_{i}$ is related to the term $B_{j}$ in the model described by the rule base. The rule base is thus represented by the matrix $\tilde{\Phi} \in I^{n \times m}$, where $n$ and $m$ are the cardinality of fuzzy partitions associated to $\mathbf{A}(X)$ and $\mathbf{B}(Y)$. In symbolic fuzzy systems, the input description $\tilde{X}_{0}$ is computed from the input value $x_{0} \in X$ as in (2). Output description $\tilde{Y}_{0}$ is computed from input description $\tilde{X}_{0}$ and the rule base $\tilde{\Phi}$ using the fuzzy relational composition:

$$
\tilde{Y}_{0}=\tilde{X}_{0} \circ \tilde{\Phi}
$$

In this work, normalised and orthogonal fuzzy sets are considered in fuzzy partitions such that:

$$
\begin{gathered}
\forall i, \exists x_{0} \in X \quad / \quad A_{i}\left(x_{0}\right)=1, \\
\forall x, \sum_{i} A_{i}\left(x_{0}\right)=1 .
\end{gathered}
$$

As an example, suppose the variable $X$ with the descriptor set $\mathbf{A}(X)=\left\{A_{0}, A_{1}, A_{2}\right\}$, the variable $Y$ with the descriptor set $\mathbf{B}(Y)=\left\{B_{0}, B_{1}\right\}$ and the fuzzy rule base:

$$
\begin{aligned}
\tilde{A}_{0} & \rightarrow \tilde{B}_{1} \\
\tilde{A}_{1} & \rightarrow \tilde{B}_{0} \\
\tilde{A}_{2} & \rightarrow \tilde{B}_{1}
\end{aligned}
$$

The prototype description of the rule base is given by the matrix $\tilde{\Phi}$ :

\begin{tabular}{|c|c|c|c|}
\hline \multicolumn{2}{|c|}{$\tilde{\Phi}$} & \multicolumn{2}{c|}{$\mathbf{B}(Y)$} \\
\cline { 3 - 4 } \multicolumn{2}{|c|}{} & $B_{0}$ & $B_{0}$ \\
\hline \multirow{3}{*}{$\mathbf{A}(X)$} & $A_{0}$ & 0 & 1 \\
\cline { 2 - 4 } & $A_{1}$ & 1 & 0 \\
\cline { 2 - 4 } & $A_{2}$ & 0 & 1 \\
\hline
\end{tabular}

Table 1 : Knowledge base.

Values different from 0 or 1 could be used to weight the confidence to the rules. Fig. 1 gives the representation of the fuzzy relation defined by the rule base where the product has been used as t-norm and the limited sum has been used as t-conorm.

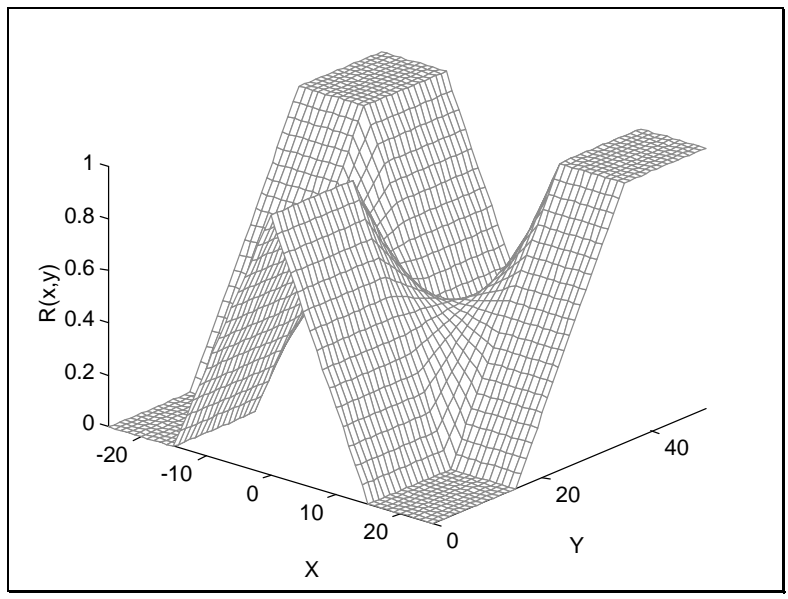

Figure 1. Fuzzy relation

Using the sum-product composition operator for symbolic fuzzy inference (4), the description $\tilde{Y}_{0}$ is easily computed for all $B_{j} \in \mathbf{B}(Y)$ as a vector matrix product:

$$
Y_{0}\left(B_{j}\right)=\sum_{1 \leq i \leq n} X_{0}\left(A_{i}\right) \Phi\left(A_{i}, B_{j}\right)
$$

If more than one variable occurs in the antecedent of the rules, the input description is defined in the cartesian product of the input descriptor sets [Babuska 96].

The fuzzy relation concept is well adapted to diagnosis task as that amounts to saying that the decision of a fault is related to prototype situation of the residuals. Between prototype situations, the fuzzy relation interpolates the conclusion.

In FDI applications, input descriptions are generally computed from numerical residuals. Each term in the output 
descriptor set is associated to a fault and output descriptions are partial conclusions on fault occurrence from information given as input. Final conclusions are computed by aggregation of partial conclusions as in multi-criteria decision making.

\subsection{Multi-criteria decision-making}

The general problem of multi-criteria decision making can be formalised as follows. Let $F_{j} \in \mathbf{F}$ the set of $M$ alternatives for solution and $C_{i} \in \mathbf{C}$ a set of $N$ criteria carrying some judgement about the occurrence of each alternative. The decision problem consists in choosing the "best alternative" $F_{0}$ from the information contained in the criteria [Zimmermann 96].

Fuzzy methods for multi-criteria decision making assume criteria as flexible constraints on the alternative set, represented by the description $\tilde{C}_{i}$. The membership function value $C_{i}\left(F_{j}\right)$ represents the judgement of alternative $F_{j}$ given by the criterion $C_{i}$.

In FDI applications, alternatives for solution are the modelled faults. In a faulty situation, the most suitable fault $F_{0}$ must be isolated from the criteria carrying symptom information. The proposed framework for FDI decision is summarised in Figure 2.

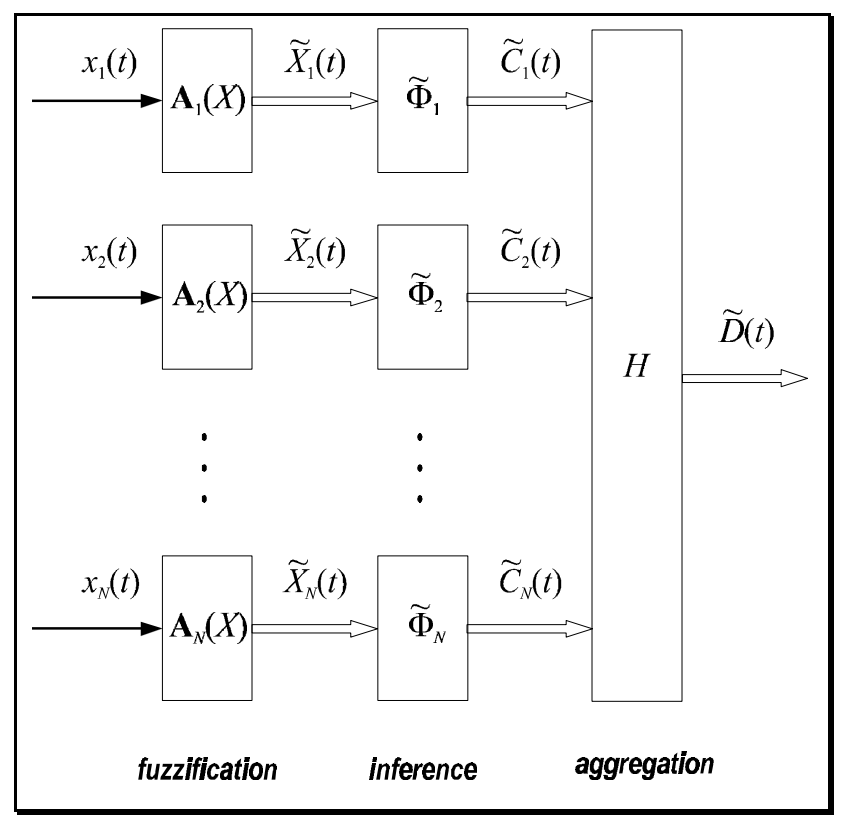

Figure 2 : The decision procedure.

The attributes $x_{i}$ represent numerical symptoms (generally residuals) whose symbolic descriptions, denoted by $\tilde{X}_{i}$, are computed by the corresponding fuzzy partition $\mathbf{A}_{i}(X)$. Each criterion $\tilde{C}_{i}$ represents a partial conclusion computed from each symptom description by symbolic fuzzy inference as:

$$
\tilde{C}_{i}=\tilde{X}_{i} \circ \tilde{\Phi}_{i}
$$

Otherwise, symbolic symptoms can be expressed directly as fuzzy descriptions $\tilde{X}_{i}$. Moreover, expert advice, represented as additional criteria $\tilde{C}_{i}$, can also be integrated in this framework.

The solution to the decision problem is carried out in two steps:

- the aggregation step, to aggregate the partial conclusions for each fault

- $\quad$ and the decision step, to decide the most suitable fault.

The aggregation step results in the fuzzy description $\tilde{D}$, defined on the alternative set $\mathbf{F}$, whose membership function is computed by:

$$
D\left(F_{j}\right)=\boldsymbol{h}\left(C_{1}\left(F_{j}\right), \ldots, C_{N}\left(F_{j}\right)\right), \forall F_{j} \in \mathbf{F}
$$

where $\boldsymbol{h}:[0,1]^{N} \rightarrow[0,1]$ is an aggregation operator, whose properties depend on the semantics in a given application: a conjunctive aggregation means that all criteria must be satisfied while a disjunctive aggregation means that at least one criteria must be satisfied.

The final decision $F_{0}$ is computed by a decision rule, usually the maximum rule:

$$
F_{0} / D\left(F_{0}\right)=\max _{\forall F_{j} \in \mathbf{F}}\left[D\left(F_{j}\right)\right]
$$

Two approaches for the design of the proposed decision procedure are presented in next section. In the knowledge based approach the symbolic rule base weights $\Phi\left(A_{\mathrm{i}}, B_{\mathrm{j}}\right)$ are derived either from the model structure if it is available or from expert knowledge if it exists. In the supervised learning approach, the rule base weights are learned from experimental data.

\section{The decision procedure design}

\subsection{The knowledge based approach}

A fuzzy partition is defined for each residual from expert advice. The symbolic terms of each fuzzy partition are defined in order to represent qualitative values for the description of the corresponding symptom. The symbolic symptom values generalise the numerical values whose fuzzy descriptions are computed from the corresponding fuzzy partition. 
For each residual the knowledge is translated into tables as Table 2. In such a table, the rows are associated to the symbolic terms in the symptom's fuzzy partition and the columns are associated to the faults $F_{j} \in \mathbf{F}$. The values in the table represent the influence of each symbolic value in the occurrence of each fault.

\begin{tabular}{|c|c|c|c|}
\hline$\tilde{\Phi}_{i}$ & $F_{1}$ & $\cdots$ & $F_{M}$ \\
\hline$A_{i 1}$ & $\Phi_{i}\left(A_{i 1}, F_{1}\right)$ & $\cdots$ & $\Phi_{i}\left(A_{i 1}, F_{M}\right)$ \\
\hline$\vdots$ & $\vdots$ & $\ddots$ & $\vdots$ \\
\hline$A_{i i_{i}}$ & $\Phi_{i}\left(A_{i i_{i}}, F_{1}\right)$ & $\cdots$ & $\Phi_{i}\left(A_{i i_{i}}, F_{M}\right)$ \\
\hline
\end{tabular}

Table 2 : Knowledge base.

The weights are defined using the model structure and the residual sensitivity to the fault. As an example, let us suppose that the residual is described by three symbolic terms $\{Z, M, N Z\}$; where $Z$ means zero, $N Z$ means that the residual is clearly positive or negative, and $M$ means that the residual is slightly affected. When a residual is less sensitive to fault $F_{1}$ than to fault $F_{2}$, the weights related to symbolic terms $\{Z, M, N Z\}$ are chosen as follows:

- $\quad N Z$ : the two faults may have occurred thus the two weights are chosen equal to 1 . In this case, the knowledge derives directly from the model structure.

- $Z$ : neither fault has occurred so the two weights are chosen equal to 0 . In this case, the knowledge derives directly from the model structure too.

- $\quad M$ : the weights are chosen according to the residual sensitivity to the faults. A high sensitivity of the residual to $F_{2}$ leads to a low confidence in the occurrence of the fault because a small fault can lead to a residual value $M$, thus the weight of the rule relative to $F_{2}$ is chosen close to 0 . In contrary if the residual is less sensitive to the alternative $\left(F_{1}\right)$, the confidence to the fault occurrence is high. The weight is then chosen close to 1 . Table 3 shows an example. In this case the knowledge is derived from the sensitivity analysis.

\begin{tabular}{|c|c|c|}
\hline$\tilde{\Phi}_{i}$ & $F_{1}$ & $F_{2}$ \\
\hline$Z$ & 0 & 0 \\
\hline $\boldsymbol{M}$ & $\mathbf{0 . 8}$ & $\mathbf{0 . 2}$ \\
\hline$N Z$ & 1 & 1 \\
\hline
\end{tabular}

Table 3 : Knowledge base example.

The table associated to each symptom is used as rule weights of the symbolic rule base used to compute partial conclusions by fuzzy inference (8).
Expert knowledge linked to the residual sensitivity to the fault may be difficult to acquire if the system analytical model is unknown or very imprecise. Then the weights of the knowledge base can be computed in a supervised learning approach.

\subsection{Supervised learning approach}

Consider a data set $S$ where each sample $s \in S$ is composed by a pair $\left\{x_{s}, \varphi_{s}\right\}$, where $x_{s}$ is the attribute vector of each residual and $\varphi_{s}$ represents its membership to each fault $F_{j} \in \mathbf{F}$. In model based FDI of dynamical systems, each sample $s \in S$ represents a time instant and the attribute vector is the residual vector. In the following, symbolic symptoms are not considered.

Fuzzy partitions are defined as in the knowledge based approach for each residual in order to compute residual descriptions. In the supervised learning approach, rule base weights can be estimated by solving a least square optimisation problem.

Consider the matrix $\mathbf{U}_{i}$, where each line contains the vector $\left[\tilde{X}_{s}\right]_{i}$ representing the description of residual $i$ corresponding to a time sample $s \in S$. The corresponding rule weight matrix $\tilde{\Phi}_{i}$ can be estimated by solving the following linear system of equations:

$$
\mathbf{U}_{i} \tilde{\Phi}_{i}=\mathbf{V}
$$

where the matrix $\mathbf{V}$ contains, in each line, the vector $\varphi_{s}$ representing the membership of the corresponding sample $s \in S$ to each fault $F_{j} \in \mathbf{F}$.

The rule base weights obtained by (11) are approximations and can lie outside the interval $[0,1]$. Rule weights must thus be normalised by a linear transformation.

The linear system (11) can be numerically ill-conditioned. By analogy to some neural networks [Evsukoff 98], residual descriptions in the data set can be concatenated to form the matrix $\mathbf{Z}=\left[\begin{array}{lll}\mathbf{U}_{1} & \cdots & \mathbf{U}_{N}\end{array}\right]$. The augmented matrix weights $\tilde{\Theta}$ can thus be estimated by solving the following linear system:

$$
\mathbf{Z} \tilde{\Theta}=\mathbf{V}
$$

The rule base weights for each attribute can be computed from the result of (12). By multiplying both sides of (12) by $\mathbf{V}^{*}$, the pseudo inverse of $\mathbf{V}$ :

$$
\left[\begin{array}{lll}
\mathbf{V}^{*} \mathbf{U}_{1} & \cdots & \mathbf{V}^{*} \mathbf{U}_{N}
\end{array}\right] \widetilde{\Theta}=\mathbf{1}
$$

where $\mathbf{1}$ is the corresponding identity matrix. 
By multiplying both sides of (11) by $\mathbf{V}^{*}$ :

$$
\left[\mathbf{V}^{*} \mathbf{U}_{i}\right] \tilde{\Phi}_{i}=\mathbf{1}
$$

From (14), (13) can be rewritten as:

$$
\left[\begin{array}{lll}
\tilde{\Phi}_{1}^{*} & \cdots & \tilde{\Phi}_{N}^{*}
\end{array}\right] \tilde{\Theta}=\mathbf{1}
$$

where $\tilde{\Phi}_{i}^{*}$ is the pseudo inverse of $\tilde{\Phi}_{i}$. The rule base weights $\widetilde{\Phi}_{i}$ for each attribute can thus be computed directly from $\tilde{\Theta}$ by solving (15).

\section{The application}

The decision approaches presented above are applied in FDI of a multi-input multi-output system: a winding machine sketched in Figure 3.

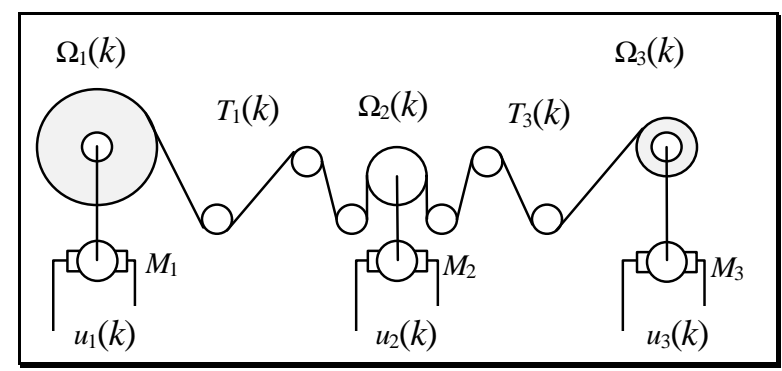

Figure 3 : The winding machine.

The system is composed of three electric motors $M_{1}, M_{2}$ and $\mathrm{M}_{3}$, controlled by the input vector $\mathbf{u}(k)=\left[\begin{array}{lll}u_{1}(k) & u_{2}(k) & u_{3}(k)\end{array}\right]^{\mathrm{T}}$. The measured outputs $\mathbf{y}(k)=\left[\begin{array}{lll}T_{1}(k) & \Omega_{2}(k) & T_{3}(k)\end{array}\right]^{\mathrm{T}}$ represent respectively the measured strip tensions, and the speed developed by the motor $\mathrm{M}_{2}$.

A model of this system has been identified by standard identification techniques [Weber 98]. The output error parity equation based on such model can be written as:

$$
\begin{aligned}
\mathbf{r}(k) & =\mathbf{y}(k)-\hat{\mathbf{G}}(q) \mathbf{u}(k) \\
& =\left[\begin{array}{c}
T_{1}(k) \\
\Omega_{2}(k) \\
T_{3}(k)
\end{array}\right]-\left[\begin{array}{ccc}
G_{11}(q) & G_{12}(q) & 0 \\
0 & G_{22}(q) & 0 \\
0 & G_{32}(q) & G_{33}(q)
\end{array}\right]\left[\begin{array}{l}
u_{1}(k) \\
u_{2}(k) \\
u_{3}(k)
\end{array}\right]
\end{aligned}
$$

where $\mathbf{r}(k)$ is the residual vector and $\hat{\mathbf{G}}(q)$ is the identified model.

Only sensor and actuator faults are considered. The faults are represented by the set $\mathbf{F}=\left\{F_{1}, \ldots, F_{6}\right\}$, where $\left\{F_{1}, F_{2}, F_{3}\right\}$ correspond to sensor faults and $\left\{F_{4}, F_{5}, F_{6}\right\}$ correspond to actuator faults. The incidence matrix [Gertler 90] derived from the model (16) is written as:

$$
\Xi=\left[\begin{array}{llllll}
1 & 0 & 0 & 1 & 1 & 0 \\
0 & 1 & 0 & 0 & 1 & 0 \\
0 & 0 & 1 & 0 & 1 & 1
\end{array}\right]
$$

where lines are associated to residuals and columns are associated respectively to sensor and actuator faults.

\section{a) Knowledge derived from the model structure:}

A normalised and orthogonal fuzzy partition is defined for each residual in order to compute residual descriptions by the symbolic terms $\{Z, N Z\}$, where $Z$ means that the residual is zero and $N Z$ means that the residual is positive or negative.

In the knowledge-based approach, the rule base for each residual is derived directly from the incidence matrix (17) by taking lines as the influence of $N Z$ residuals to faults occurrence. The influence of $Z$ residuals to faults occurrence are the complement of the influence of $N Z$ residuals, such that the rule weight matrices are given by:

$$
\begin{aligned}
& \tilde{\Phi}_{1}=\left[\begin{array}{llllll}
0 & 1 & 1 & 0 & 0 & 1 \\
1 & 0 & 0 & 1 & 1 & 0
\end{array}\right] \\
& \tilde{\Phi}_{2}=\left[\begin{array}{llllll}
1 & 0 & 1 & 1 & 0 & 1 \\
0 & 1 & 0 & 0 & 1 & 0
\end{array}\right] \\
& \tilde{\Phi}_{3}=\left[\begin{array}{llllll}
1 & 1 & 0 & 1 & 0 & 0 \\
0 & 0 & 1 & 0 & 1 & 1
\end{array}\right]
\end{aligned}
$$

From the incidence matrix (17), it can be seen that a sensor fault $\Delta T_{1}(k)$ cannot be distinguished from an actuator fault $\Delta u_{1}(k)$ as so as for $\Delta T_{3}(k)$ and $\Delta u_{3}(k):$ the first column is equal to the fourth column as so as for the third and sixth columns.

\section{b) Knowledge derived from the residual sensitivities:}

The residuals computed in (16) allows to use the static gain of the parallel models $\hat{\mathbf{G}}(q)$ to define the residual sensitivities:

$$
\left.\hat{\mathbf{G}}(q)\right|_{q=1}=\left[\begin{array}{ccc}
-2.78 & 0.29 & 0 \\
0 & 1.1 & 0 \\
0 & -0.18 & 1.05
\end{array}\right]
$$

and the residual sensitivities to sensor faults are equal to 1 .

The knowledge base is written using three symbolic terms $\{Z, M, N Z\}$. 


$$
\begin{aligned}
& \tilde{\Phi}_{1}=\left[\begin{array}{cccccc}
0 & 1 & 1 & 0 & 0 & 1 \\
0.2 & 0 & 0 & 0.2 & 0.8 & 0 \\
1 & 0 & 0 & 1 & 1 & 0
\end{array}\right] \\
& \tilde{\Phi}_{2}=\left[\begin{array}{cccccc}
1 & 0 & 1 & 1 & 0 & 1 \\
0 & 0.2 & 0 & 0 & 0.2 & 0 \\
0 & 1 & 0 & 0 & 1 & 0
\end{array}\right] \\
& \tilde{\Phi}_{3}=\left[\begin{array}{cccccc}
1 & 1 & 0 & 1 & 0 & 0 \\
0 & 0 & 0.2 & 0 & 0.8 & 0.2 \\
0 & 0 & 1 & 0 & 1 & 1
\end{array}\right]
\end{aligned}
$$

\section{c) Knowledge derived from data:}

A data set generated by simulation of each one of the modelled faults is used to compute estimations of the rule weight matrices. The results obtained by solving the rule base weights for each input (eq. (11)) are the following:

$$
\begin{aligned}
\tilde{\Phi}_{1} & =\left[\begin{array}{llllll}
0.00 & 0.08 & 0.08 & 0.00 & 0.00 & 0.08 \\
0.36 & 0.00 & 0.00 & 0.32 & 0.33 & 0.00
\end{array}\right] \\
\tilde{\Phi}_{2} & =\left[\begin{array}{llllll}
0.07 & 0.01 & 0.07 & 0.07 & 0.00 & 0.07 \\
0.00 & 0.47 & 0.00 & 0.00 & 0.62 & 0.00
\end{array}\right] \\
\tilde{\Phi}_{3} & =\left[\begin{array}{llllll}
0.07 & 0.07 & 0.00 & 0.07 & 0.00 & 0.03 \\
0.00 & 0.00 & 0.68 & 0.00 & 0.07 & 0.33
\end{array}\right]
\end{aligned}
$$

By the concatenation of input descriptions as (12) the solution of the system (15) is given by:

$$
\begin{aligned}
& \tilde{\Phi}_{1}=\left[\begin{array}{llllrl}
0.02 & 0.07 & 0.03 & 0.02 & -0.06 & 0.04 \\
0.32 & 0.00 & 0.00 & 0.32 & 0.31 & 0.00
\end{array}\right] \\
& \tilde{\Phi}_{2}=\left[\begin{array}{llllrl}
0.03 & 0.05 & 0.03 & 0.03 & -0.05 & 0.03 \\
0.00 & 0.50 & 0.00 & 0.00 & 0.50 & 0.00
\end{array}\right] \\
& \tilde{\Phi}_{3}=\left[\begin{array}{llllrl}
0.04 & 0.07 & 0.00 & 0.04 & -0.06 & 0.01 \\
0.00 & 0.00 & 0.30 & 0.00 & 0.22 & 0.30
\end{array}\right]
\end{aligned}
$$

It can be seen that both rule-based weights computed by supervised learning approach reduce to the ones obtained by the knowledge-based approach after normalisation. Also, the first column weights is very close to the forth column weights as so as for the third and sixth columns.

\section{Conclusion}

This work has presented two approaches to derive decision procedures for FDI application. In the knowledge-based approach, fuzzy rules are defined directly from model structure represented as an incidence matrix, and the rules weights are defined using symptom sensitivities. In the supervised learning approach, the model structure can be derived from a data set. The symbolic to numeric integration provided by the proposed approach allows to integrate easily symbolic symptoms into the decision procedure. The unified representation adopted here allows the cohabitation of the knowledge based approach and the supervised learning approach within the same decision procedure. The application to the winding machine shows that the supervised learning approach achieves the correct model structure. This approach can be used to derive automatically decision procedures for FDI when black box models are used.

\section{References}

[Babuska 96] Babuska R., Verbruggen H. B., "An overview of fuzzy modelling for control", Control Eng. Practice, 4 (11), pp. 1593-1606, (1996).

[Cassar 92] Cassar J. P., Starowiecki M. Ferhati R., "Multivalued logic voting scheme for residual evaluation in failure detection and isolation system", IFAC/IFIP/IMACS Symposium on Artificial Intelligence in Real-Time Control, Delft, The Netherland, (1992).

[Evsukoff 98] Evsukoff A, Gentil S., "Symbolic-numeric integration in pattern recognition", Proc. European Workshop of Fuzzy Decision Analysis and Neural Networks-EFDAN'98, (1998).

[Frank 94] Frank P.M.," Application of Fuzzy Logic to Process Supervision and Fault Diagnosis", IFAC SAFEPROCESS'94, 2, (1994).

[Foulloy 95] Foulloy L., Galichet S., "Typology of fuzzy controllers - in Theoretical Aspects of Fuzzy Control", Eds. H. Nguyen, M. Sugeno, R. Tong and R. Yager, J. Wiley Publishers, (1995).

[Gertler 90] Gertler J., Singer D., "A new structural framework for parity equation based failure detection and isolation", Automatica, 26 (2), pp. 381-388, (1990).

[Gertler 92] Gertler J., Anderson K. C., "An evidential reasoning extension to qualitative model-based failure diagnosis", IEEE Trans. on Systems Man Cybernetics 22 (2), pp. 275-288, (1992).

[Isermann 97] Isermann R., "Supervision, fault-detection and fault-diagnosis methods - an introduction", Control Eng. Practice, 5 (5), pp. 639-652, (1997).

[Isermann 98] Isermann R., "On fuzzy logic applications for automatic control, supervision and fault diagnosis", IEEE Trans. on Systems Man Cybernetics - Part A: Systems and Humans, 28 (2), pp. 221-234, (1998).

[Kosko 92] Kosko B., "Neural Networks and Fuzzy Systems", Prentice Hall, (1992).

[Montmain 91a] Montmain J., Gentil S., "Qualitative event analysis for fault diagnosis", IFAC SAFEPROCESS'91, Baden-Baden, (1991).

[Weber 98] Weber P., Gentil S., "Fault detection and isolation using multiple model parameter estimation", IMACS, Multiconference, Computational Engineering in Sytems applications, Nabeul-Hammamet, Tunisia, (1998).

[Zimmermann 96] Zimmermann H.-J., "Fuzzy Set Theory and its Applications", Kluwer, (1996). 